\title{
Desarrollo de una Actividad de Aprendizaje Experiencial para el aprendizaje de planes de control de la calidad.
}

\author{
Oscar Trull-Domínguez ${ }^{a}$, Ángel Peiró-Signes ${ }^{b}$, Marival Segarra-Oña ${ }^{c}$ \\ ${ }^{a}$ Departamento de Estadística e Investigación Operativa Aplicada y Calidad (Universitat Politècnica \\ de València, otrull@eio.upv.es), b Departamento de Organización de Empresas (Universitat \\ Politècnica de València, anpeisig@omp.upv.es) y ${ }^{\mathrm{c}}$ Departamento de Organización de Empresas \\ (Universitat Politècnica de València, maseo@omp.upv.es).
}

\begin{abstract}
This paper develops an Experiential Learning Activity (ELA) that focuses on the learning of concepts, procedures and documents necessary for the creation and effective use of control plans for quality assurance. The activity immerses the student in an environment of play and competition that allows the students to enjoy adequate motivation and concentration for the teachinglearning process. The activity has specially designed catapults, with which the students must perform three phases: assembly of them, learning to use and use in context, competing with their peers. The learning itinerary begins with experimentation, to move on to understanding and finally to creation and design.
\end{abstract}

Keywords: Experiential Learning Activities, control plan, quality, quality assurance.

\begin{abstract}
Resumen
Este artículo desarrolla una Actividad de Aprendizaje Experimental (ELA) que permite el aprendizaje de los conceptos, procedimientos y documentos necesarios para la creación y uso efectivo de planes de control de aseguramiento de la calidad. La actividad sumerge al alumno en un ambiente de juego y competición que permite al alumno disfrutar de la motivación y concentración adecuadas para el proceso de enseñanzaaprendizaje. La actividad dispone de unas catapultas especialmente diseñadas, con las que los alumnos deben realizar tres fases: ensamblaje de las mismas, aprendizaje de uso y utilización en contexto, compitiendo con sus compañeros. El itinerario del aprendizaje empieza con la experimentación, para pasar a la comprensión y finalmente a la creación y diseño.
\end{abstract}

Palabras clave: Aprendizaje experiencial, planes de control, calidad, aseguramiento de la calidad.

\section{Introducción}

La enseñanza tradicional basa su metodología de enseñanza-aprendizaje en la secuencia básica de profesor al alumno. El profesor, mediante clases magistrales, muestra una serie de conceptos y procedimientos que el alumno debe comprender para su ejercicio posterior 
Desarrollo de una Actividad de Aprendizaje Experiencial para el aprendizaje de planes de control de la calidad.

mediante una serie de prácticas. Esta metodología es válida en la mayoría de los entornos académicos. Sin embargo, la enseñanza de conceptos de calidad requiere de un particular interés por parte del profesor. Es importante resaltar que, si el concepto calidad resulta complicado de asimilar por gran parte del personal con experiencia laboral, este concepto resulta realmente complicado para aquellos alumnos sin esa experiencia. Lo mismo sucede con las herramientas de la calidad. Resulta prácticamente imposible dar a conocer conceptos y herramientas sofisticadas a algunos menos que no disponen de experiencia previa.

Según los estudios recientes sobre motivación del alumnado y su capacidad de adquisición de conceptos, se indica que el fundamento del proceso de enseñanza-aprendizaje debe recaer sobre el estudiante. Esto sugiere un cambio de perspectiva en la enseñanza. Los procesos habituales han sido orientados con el flujo de la formación desde el profesor hacia el alumno y una retroalimentación en las evaluaciones. Sin embargo, con este nuevo enfoque, el alumno es el que transmite la necesidad de aprendizaje y el formador evalúa constantemente las necesidades del alumno guiándole en una dirección efectiva hacia el aprendizaje. Pero llevar a cabo esta metodología no es inmediata. Es por ello, que las actividades de aprendizaje experiencial (ELA) se constituyen como una potente herramienta de aprendizaje (Peiró-Signes et al., 2017a and 2017b, Trull-Domínguez et al., 2017). Este tipo de técnicas tienen una serie de características inherentes al proceso:

- Aprendizaje deductivo de los conceptos. El alumno adquiere conceptos partiendo de la experiencia, viendo su utilidad y posteriormente comprendiendo los conceptos. A partir de ahí, al alumno será capaz de generar nuevas situaciones donde aplicar los conceptos.

- Aplicabilidad. Los conceptos se adquieren a través de la experiencia en diferentes aplicaciones. Ello provoca una abstracción de la aplicación y una generalización del problema. Internamente, el alumno está desarrollando el aprendizaje de forma consciente y estructurada.

- Escalabilidad. Los problemas prácticos pueden Disponerse de forma escalable. Así, el alumno refuerza contenidos ante la adquisición de nuevos conceptos.

- Motivación. El hecho de utilizar actividades lúdicas provoca una motivación en el alumnado que de otro modo difícilmente se podría conseguir.

Todas estas características tienen como resultado una ELA sofisticada. La planificación de estas actividades es meticulosa y debe ser en parte retroalimentada y revisada en cada nueva situación. Finalmente, la ejecución de la ELA debe estar limitada en el tiempo y bajo una estricta supervisión por parte del formador.

En este artículo se presenta una actividad experiencial dirigida a estudiantes de ingeniería en los cursos finales de su formación. En ella se tratan conceptos de calidad previamente adquiridos, y se desarrolla el concepto de plan de control de calidad o de aseguramiento de la calidad. Para que la actividad tenga un mayor éxito experiencial, se utilizará un juego de catapultas que los alumnos utilizarán para sus pruebas y finalmente habrá un desafío (elemento altamente motivador) con el que poner en práctica los conocimientos adquiridos. Esta actividad está organizada en dos sesiones de dos horas.

(cc) EY-NC-ND 2018, Universitat Politècnica de València

Congreso IN-RED (2018) 


\section{Objetivos}

El principal objetivo de la actividad experiencial de aprendizaje (ELA) desarrollada en este artículo consiste en aprender técnicas básicas del aseguramiento de la calidad. La ELA desarrolla el concepto de Planes de Control (CP) así como el Análisis Modal de Fallos y Efectos (FMEA), con el trabajo realizado por los alumnos cuando revisan los procesos de ensamblado de la catapulta, identificando los posibles errores, causas y efectos. Con ello, el alumno entiende los errores cometidos, y cómo un plan de control ayuda a evitarlos, sobretodo en un ambiente industrial plagado de procesos. Adicionalmente, se desarrollan actividades transversales, como es el trabajo cooperativo, en equipo, comunicación, etc.

(LaForge y Busing, 1998), indican los pasos a seguir para realizar una actividad experiencial, organizado en fases: una primera fase de planificación, una de desarrollo de la actividad y una última de toma de datos y retroalimentación para la mejora de la actividad.

En la fase de planificación, el formador debe emplear su experiencia para desarrollar una actividad que pueda resultar lo más veraz y cercana a la actividad industrial, teniendo en cuenta el nivel de conocimientos de los alumnos, y los objetivos a desarrollar (Harpeln and Hakel, 2013). Se trata, sin duda, de la fase más compleja. Hay que tener en cuenta, además, que, en esta actividad, el estudiante debe tener la capacidad de reflexionar sobre sus decisiones y de poder ejecutar él conocimiento adquirido. Es fundamental para una motivación positiva (Kold, 2014, Wheeler and McLeod, 2002).

En la fase de introducción, el instructor explica los elementos básicos de la experiencia, objetivos, organización y recursos utilizados, así como la reglas a observar durante el desarrollo de la actividad.

En la fase de desarrollo, del estudio ante debe estar relajado y motivado. Esta actitud permite de forma inconsciente una mejor atención al desarrollo de la actividad y consigue que el alumno esté inmerso en la actividad. Realizar actividades sin una motivación plena pude motivar una pérdida constante de atención, independientemente del nivel del alumnado. Sin embargo, en esta dase, el formador debe trabajar con la atención plena del alumnado. Debe estar atento para que el alumnado no divague en la actividad y pierda el fundamento. No hay que olvidar tampoco, que, en la ejecución de la actividad, aspectos transversales de la formación también se deben desarrollar, como el trabajo en equipo, proactividad, respeto a las decisiones ajenas, etc.

Finalmente, en la fase de recopilación, es necesario observar el nivel de adquisición de conceptos realizados por los alumnos, así como la percepción de los conceptos menos del resultado de la actividad.

\section{Desarrollo de la innovación.}

\subsection{Planificación}

La planificación se realiza teniendo en cuenta los aspectos fundamentales de distribución del tiempo, espacios y alumnos. Como las actitudes transversales son de especial 
Desarrollo de una Actividad de Aprendizaje Experiencial para el aprendizaje de planes de control de la calidad.

importancia, los alumnos se organizarán en grupos. La actividad grupal permite el desarrollo de las lluvias de ideas, trabajo en equipo y resolución de problemas.

Uno de los aspectos principales es la conexión del problema con el mundo real. Esta es la base que consolida el aprendizaje. La motivación forma parte del engranaje que debe hacer funcionar toda la actividad, que junto con una formación práctica deben conseguir que la actividad se desarrolle de forma funcional y eficiente.

Por lo tanto, se organiza la actividad del siguiente modo:

1. Una primera fase en la que disponen de material y procedimientos a seguir. Esta es una fase puramente procedimental. Conviven con el concepto de planes de control.

2. Una fase de deducción. Qué es lo que han hecho y la utilidad en la industria.

3. Aplicación y desarrollo los alumnos crean un nuevo plan de control aplicado a una nueva situación.

4. Recapitulación. El final de la práctica debe recoger todos los aspectos adquiridos y valorarlos. El formador aporta mejoras y se pone en común los diferentes planes realizados por los grupos para entablar una discusión y fortalecer los conocimientos adquiridos.

Un elemento diferenciador de esta actividad es el uso de catapultas y el realizar un reto entre los alumnos para vencer unos a otros. Está claro que la rivalidad no es un gran valor, pero sin duda es capaz de activar realmente la motivación del alumnado.

\subsection{Introducción.}

Para conseguir una completa motivación del alumnado, es importante comenzar la actividad con un elemento sorpresa que cree expectación. El instructor llevará el material en un maletín o lo tendrá preparado antes de la llegada de los estudiantes. Mostrará una catapulta completamente montada y discutirá con los alumnos cómo montarla de forma eficiente y sin cometer errores. Tras unos minutos de discusión, el formador dividirá a los estudiantes en grupos de tres, o en caso necesario, en grupos de cuatro. Se les dará el material necesario, y entonces se explica el objetivo inicial. Esta introducción durará alrededor de media hora. El material mínimo necesario constará de:

- Un juego completo de catapulta y herramientas.

- Un checklist con los materiales suministrados.

- Unas instrucciones de montaje.

- Un plan de control para el montaje.

(c) EY-NC-ND 2018, Universitat Politècnica de València

Congreso IN-RED (2018) 


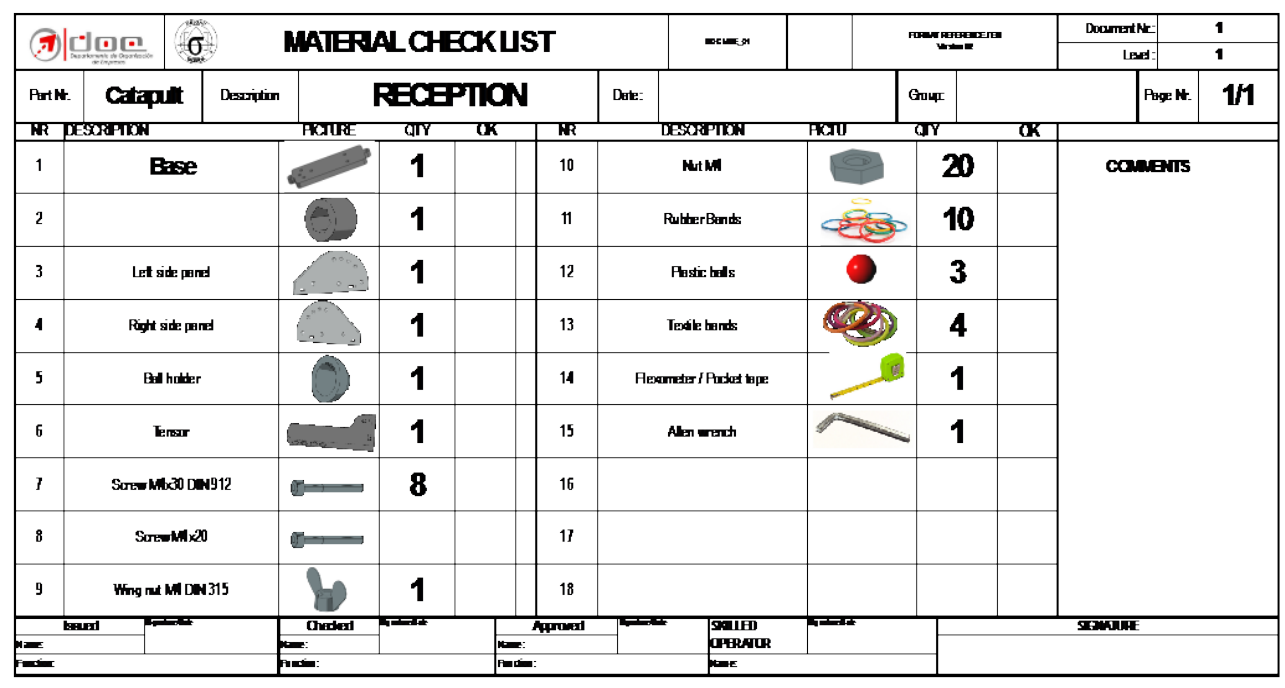

Fig 1. Lista de comprobación del material para la realización de la práctica.

\subsection{Actividad}

Para la correcta organización del equipo, uno de los componentes debe montar la catapulta, mientras que otro debe leer las instrucciones y otro seguir el plan de control. En caso de haber un alumno más, las tareas de controlar las instrucciones y la asesorar el montaje puede dividirse entre dos componentes.

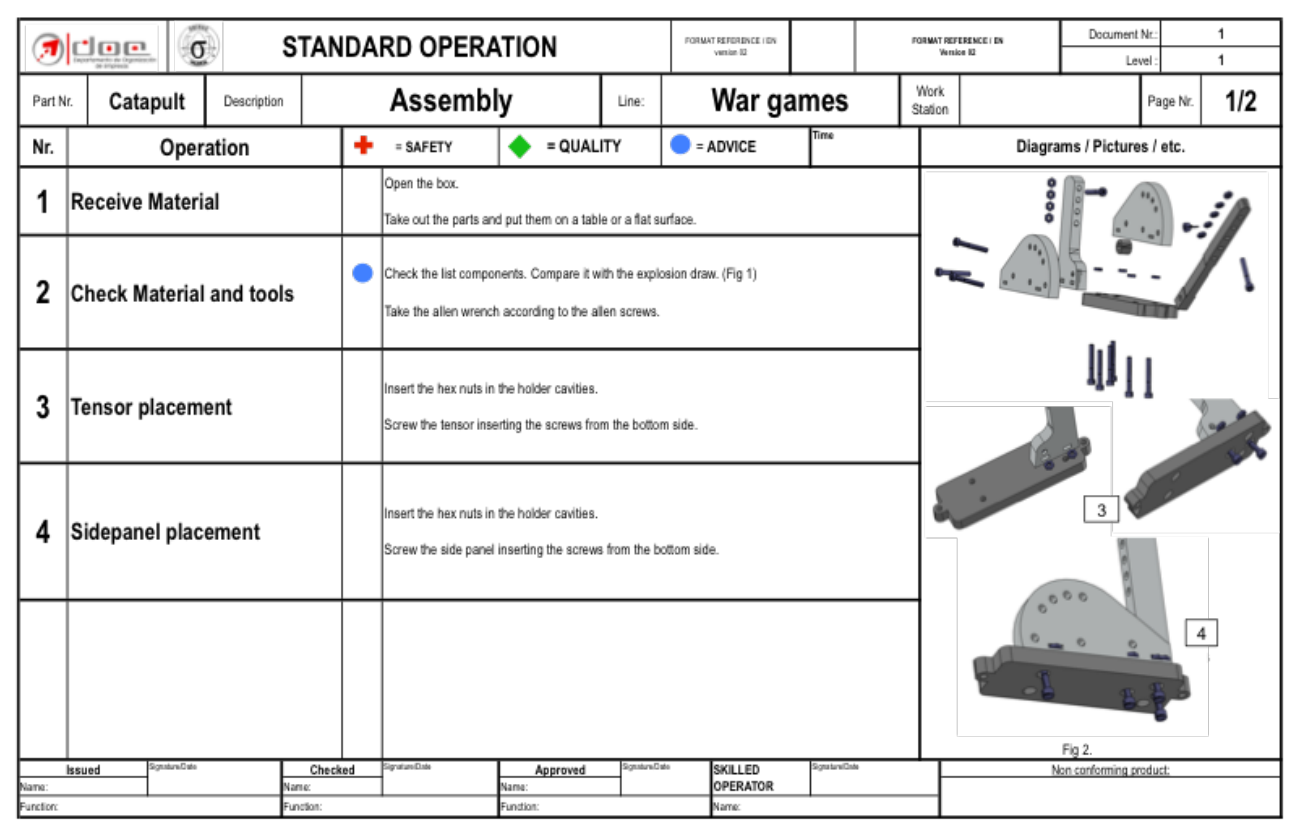

Fig 2. Operación estándar de montaje de la catapulta.

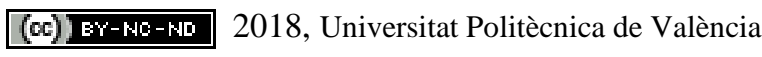
Congreso In-Red (2018) 
Desarrollo de una Actividad de Aprendizaje Experiencial para el aprendizaje de planes de control de la calidad.

Los grupos montan las catapultas. Deben controlar el tiempo de montaje y los fallos que realizan para posteriormente realizar el debate. Si es posible realizarlo, es conveniente introducir dificultades con piezas mal indicadas, que falten, o con alguna instrucción no muy clara. Así se puede poner en práctica el plan de control.

Una vez terminado, se debate sobre las actividades realizadas. Su conveniencia, ventajas y desventajas deben ser abordados en el debate. Por último, el formador debe exponer un problema real de la industria o servicios y discutir sobre esta herramienta.

Este debate debe ser aprovechado por el formador para explicar los diferentes aspectos del plan de control, así como la parte práctica para realizar un plan de control. Esta actividad debe durar entre cuarenta y cincuenta minutos.

A los alumnos se les informa de los nuevos objetivos: deben realizar un combate entre ellos para ganar destruyendo al ejército enemigo. El juego consiste en disponer cada grupo de cinco muñecos de pequeño tamaño que se disponen en formación triangular y aúna distancia determinada, de modo que alternen distancias cortas de casi 1,5 metros hasta más largas como 3 metros. Estas distancias han de ser medidas y anotadas. Los componentes del grupo realizan hasta un máximo de tres lanzamientos y anotan el número de muñecos abatidos en cada lanzamiento. Los muñecos abatidos no se levantan hasta el siguiente lanzador. El juego se organiza por turnos, y cada vez ha de lanzar un equipo, y dentro de cada equipo, un integrante. Los turnos dentro de cada equipo han de ser rodados, de modo que todos los integrantes lancen aproximadamente el mismo número de lanzamientos. Los lanzamientos se organizan por pares: dos grupos hasta que se haya completado una ronda con todos los lanzadores. Luego se intercambian grupos y así sucesivamente mientras el tiempo destinado a la actividad pueda realizarse.

Esta actividad durará 20 minutos, y en el proceso los alumnos deben anotar las incidencias que observan, así como las actuaciones positivas que llevan al abatimiento de muñecos contrarios. Es importante recalcar esta última indicación porque será la base para la siguiente parte de la actividad.

Terminado el tiempo, se proclama vencedor al que mayor número de muñecos haya derribado. Pero la actividad empieza a partir de ese momento. Los alumnos han estado practicando y aprendiendo el manejo de las catapultas, $\mathrm{y}$ ahora establecen el plan de control para el proceso de lanzamiento.

Con la ayuda del formador, se debe crear un plan de control para el lanzamiento de las bolas independientemente de la distancia a la que se encuentren. La secuencia se consigue del siguiente modo: primero, separar los diferentes procesos para el disparo; segundo, ver los posibles fallos y errores que se puedan prever. A partir de aquí, se proponen ya actividades: características a medir, tener en cuenta cómo se controla la característica que se está tratando, planes de acción, etc. Este plan quedará formalizado y se pondrá en común para todos los grupos. Esta fase puede durar unos 40 minutos.

(c) EY-NC-ND 2018, Universitat Politècnica de València

Congreso IN-RED (2018) 


\section{Actividad extendida}

En caso de disponer de suficiente tiempo con el alumnado, debería poderse poner en práctica el plan de control realizado. Esto consistiría en realizar de nuevo el combate anterior, pero aplicando los conocimientos adquiridos y poniendo énfasis en seguir el plan de control. Por supuesto, no hay demasiado aporte didáctico en esta fase, aunque si motivador, ya que poner en práctica el plan construido por el alumnado permite comprobar su utilidad. Esta actividad supondría entre 20 y 30 minutos.

\subsection{Debate y discusión}

Al final de la sesión se propone una discusión y un debate sobre la actividad. Es importante recabar información sobre los contenidos adquiridos, así como de la percepción del alumnado acerca del aprovechamiento de la actividad experiencial para su desarrollo formativo. La forma más práctica para realizar esta acción es entablar un debate abierto acerca de los conceptos, y posteriormente realizar un cuestionario para la evaluación de la adquisición, y otro para la percepción. Pocas preguntas y muy directas.

Las preguntas deben ser del tipo: ¿Qué logramos con un plan de control? ¿Podemos evitar los errores estableciendo un plan de control en toda la empresa? ¿Cuántos planes de control pueden ser necesarios en una empresa?

La duración de esta parte debe rellenar el tiempo necesario hasta los 120 minutos planteados.

Finalmente se propone una serie de actividades posteriores para el completo desarrollo de la actividad. Los alumnos de cada grupo deben escoger una actividad conocida y simple, y desarrollar un plan de control sencillo en el que se incluyan aspectos tales como la recepción de material, procesos operativos, entrega a cliente, y desarrollando todos los puntos del plan.

\section{Resultados}

Evaluamos los resultados de la primera ejecución de la actividad a través de una pequeña encuesta de las percepciones de los estudiantes completada después de la clase. Utilizamos un cuestionario con una escala Likert de 1 a 5 , donde 1 corresponde a totalmente en desacuerdo y 5 a totalmente de acuerdo.

A continuación, se muestran resultados obtenidos de la encuesta de percepción por parte del alumnado sobre la comprensión y adquisición de los nuevos conceptos. Las preguntas comparan la familiaridad del Alumno con los conceptos trabajados. En una primera instancia, los alumnos fueron preguntados en un test anterior a esta práctica por los conceptos, que denominamos preguntas del grupo 1 preactividad. Los datos se comparan con las mismas respuestas dadas por los alumnos tras la actividad.

Las figuras 1 a 4 representan los datos obtenidos para esta encuesta. En ellas se pueden apreciar cómo los resultados obtenidos tras la realización de la ELA. contribuyen a mejorar la percepción del alumno de su proceso de aprendizaje y formación. 
Desarrollo de una Actividad de Aprendizaje Experiencial para el aprendizaje de planes de control de la calidad.

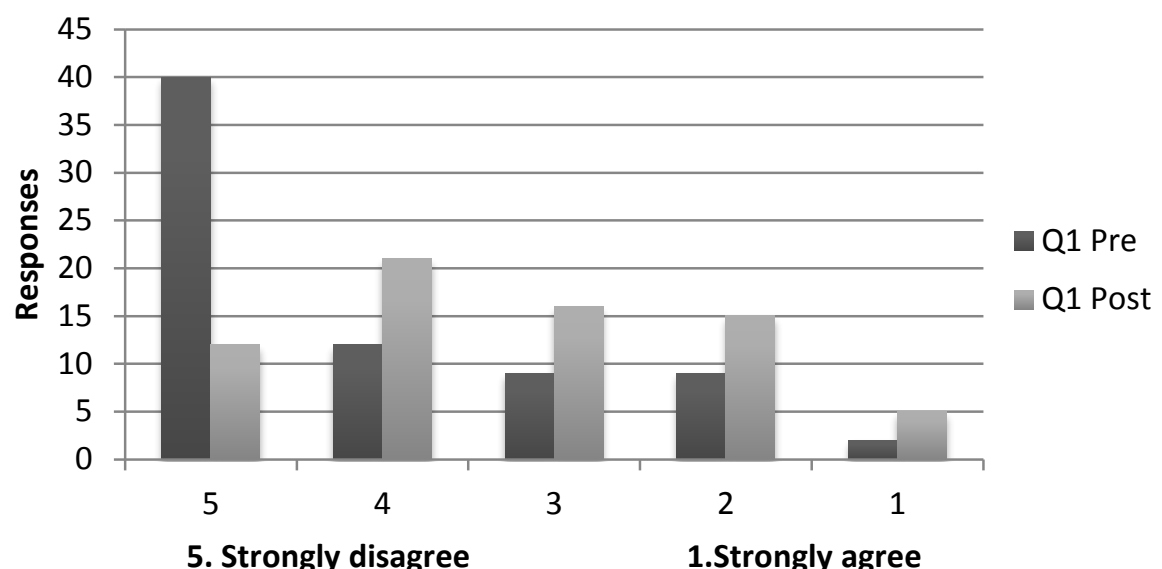

Fig 3. ¿Qué significa "Plan de Control"?. Explica su utilidad.

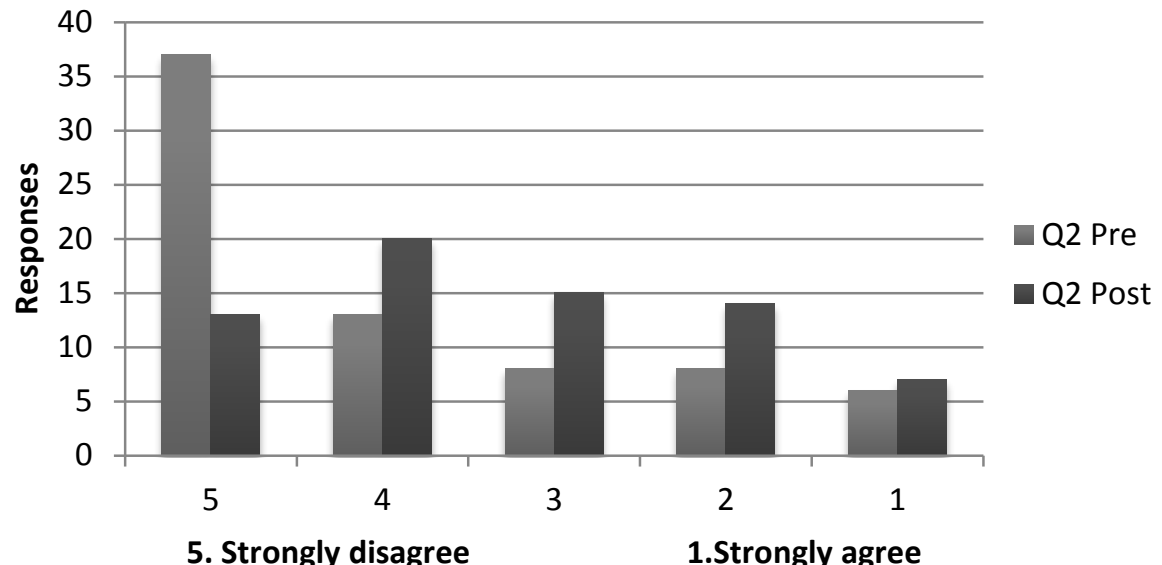

Fig. 4. Explica los requerimientos del plan de acción y cómo se asignan sus tareas.

(c) EY-NC-ND 2018, Universitat Politècnica de València

Congreso IN-RED (2018) 


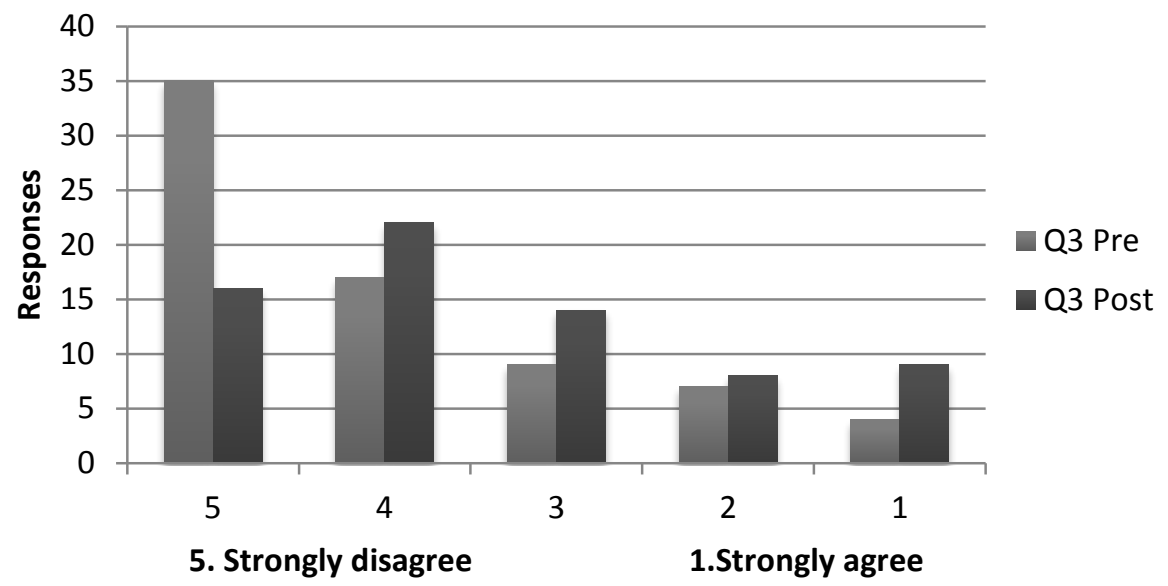

Fig. 5. Explica los elementos de especificación de un plan de control.

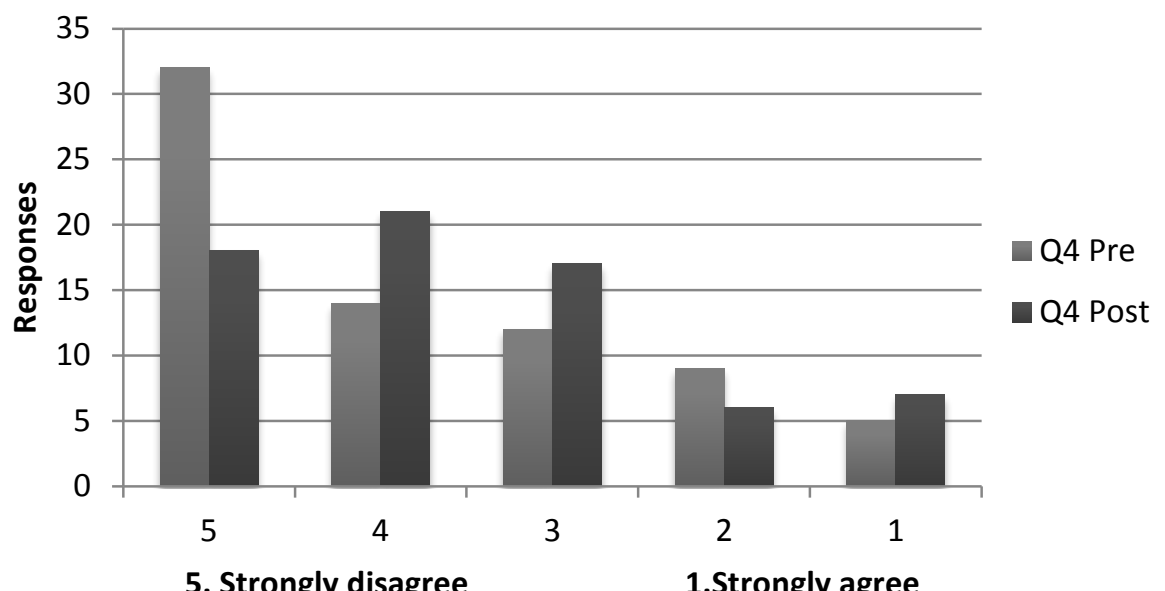

Fige 6. Describe los métodos necesarios en un plan de control.

\section{Conclusiones}

Este artículo describe una actividad experiencial basada en el uso de catapultas para introducir conceptos complejos de calidad de forma sencilla y motivadora, superando la metodología tradicional de enseñanza basada en explicaciones.

Se describe la problemática de la enseñanza de la calidad, y como las ELA pueden jugar un papel Importante en la innovación del Proceso de enseñanza -aprendizaje. Se describe la activad a realizar y cómo poder evaluarla. Por último, se analizan resultados fruto de la Actividad ya realizada y testeada con alumnos. El resultado obtenido muestra una mejora 
Desarrollo de una Actividad de Aprendizaje Experiencial para el aprendizaje de planes de control de la calidad.

sustancial en la capacidad del alumno de relacionar el trabajo realizado con la calidad y profundizar en los conceptos adquiridos.

\section{Agradecimientos}

Este trabajo forma parte del estudio desarrollado por el Grupo de Aprendizaje Experiencial (GAE) creado como EICE en la Universidad Politécnica de Valencia (UPV). Los autores desean agradecer a la UPV por el apoyo a través del PIME 2018-2019 "Adaptación y desarrollo de aprendizajes experienciales al contexto de las asignaturas ".

\section{Referencias}

PEIRO-SIGNES, A., TRULL-DOMINGUEZ, O., SEGARRA-ONA, M. V. AND DE-MIGUELMOLINA, B. (2017). Using simple experiential learning activities encourage learning of operations management concepts. Proceedings of EDULEARN17 Conference, pp. 8712-8718.

PEIRO-SIGNES, A., SEGARRA-ONA, M. V., TRULL-DOMINGUEZ, O. AND DE-MIGUELMOLINA, B. (2017). Bean bags: an experiential learning activity for quality control. Proceedings of EDULEARN17 Conference, pp. 8216-8221.

TRULL-DOMINGUEZ, O., PEIRO-SIGNES, A. AND SEGARRA-ONA, M. V. (2017). Learning statistical capacity concept through an experiential learning activity. Proceedings of EDULEARN17 Conference, pp. 8797-8801.

LAFORGE, R. AND BUSING, M. (1998). The use of industrial software to create experiential learning activities in operations management courses. Prod. Oper., vol. 7, no. 3, pp. 325-334.

HALPERN,D. AND HAKEL,M. (2003). Applying the science of learning to the university and beyond: Teaching for long-term retention and transfer. Mag. High. Learn.

KOLB,D. (2014). Experiential learning: Experience as the source of learning and development. Pearson Education, Inc.

WHEELER, J. AND MCLEOD, P. (2002). Expanding our teaching effectiveness: Understanding our responses to 'in-the-moment' classroom events. J. Manag. Educ.

(cc) EY-NC-ND 2018, Universitat Politècnica de València

Congreso IN-RED (2018) 